\title{
MULHERES EM TRATAMENTO HEMODIALÍTICO: QUALIDADE DE VIDA E ASSISTÊNCIA DE ENFERMAGEM
}

\author{
WOMEN SUBMITTED TO HEMODIALYSIS TREATMENT: \\ LIFE QUALITY AND NURSING CARE
}

\section{Lágila Cristina Nogueira Martins ${ }^{\mathrm{a}^{*}}$, Patrícia Magnabosco ${ }^{\mathrm{b}^{*}}$, Maria Elizabeth Roza Pereira $^{\mathrm{c}^{*}}$}

\author{
lagilaenfermeira@outlook.com ${ }^{\mathrm{a}}$, patriciamagnabosco@gmail.com ${ }^{\mathrm{b}}$, elizabethrz031@gmail.com ${ }^{\mathrm{c}}$
}

Universidade Federal de Uberlândia - UFU/MG*

\section{RESUMO}

Data de recebimento do artigo: 09/12/2018 Data de aceite do artigo: 18/02/2019

Introdução: A Doença Renal Crônica (DRC) é um problema de saúde pública e sua evolução relaciona-se a altas taxas de morbimortalidade no Brasil e no mundo, sendo necessário, muitas vezes, Terapia Renal Substitutiva. $\mathrm{O}$ trabalho visa avaliar a qualidade de vida $(\mathrm{QV})$ e a assistência de enfermagem de mulheres portadoras de DRC submetidas ao tratamento hemodialítico. Materiais e métodos: pesquisa descritiva, transversal com abordagem quantitativa realizada no setor de hemodiálise de um Hospital Universitário do Triângulo Mineiro. Para a coleta de dados foi utilizado um instrumento sociodemográfico semi-estruturado, SF-36 acrescido de questões referentes à assistência de enfermagem e para análise dos dados foi utilizado o programa SPSS. Resultados: das mulheres entrevistadas, 26,31\% apresentaram entre 51 e 60 anos e 57,89\% apresentaram hipertensão como doença de base da DRC. Em relação ao instrumento SF-36, os domínios aspectos físicos, aspectos emocionais e dor foram os que receberam menores escores. Quanto a assistência de enfermagem, 94,74\% das mulheres avaliaram a assistência recebida no referido local como satisfatória. Discussão: A DRC afeta principalmente pessoas em idade economicamente ativa e o tratamento debilitante interfere no desenvolvimento de atividades diárias e de lazer, gerando prejuízo psicológico, entretanto percebe-se que os escores referentes à saúde física do SF-36 afetaram mais a QV dessas mulheres do que os escores de saúde mental. Conclusão: A QV é prejudicada nesses pacientes, sendo agravada por algumas variáveis sociodemográficas, clínicas e hábitos de vida. Já a assistência de enfermagem é indispensável para o enfrentamento das dificuldades e adesão ao tratamento.

Descritores: Qualidade de vida; unidades hospitalares de hemodiálise; cuidados de enfermagem; enfermagem em nefrologia.

\section{ABSTRACT \\ Introduction: Chronic Kidney Disease (CKD) is a public health problem and its evolution is related to high morbidity and mortality rates in Brazil and in the world, often requiring Renal Renal Therapy. This study aims to evaluate the life quality and nursing care of women with chronic renal disease CKD submitted to hemodialysis treatment. Methods: descriptive, cross-sectional research with quantitative approach carried out in hemodialysis sector from a University Hospital located in the Triângulo Mineiro. In order to collect the data it was used a semi-structured socio-demographic, SF-36 complemented by matters referring to nursing care and for data analysis it was used the program SPSS. Results: of the women interviewed, 26,31\% show age between 51 and 60 years old and 57,89\% presented hypertension as the basic disease of CKD. Regarding the SF-36 instrument, the domains physical aspects, emocional aspects and pain were those that received the lower scores. As for nursing care, $94,74 \%$ of women evaluated the care received at the site as satisfactory. Dis- cussion: CKD mainly affects people of economically active age and the debilitating treatment interferes with the development of daily and leisure activities, generating psychological damage, however it is noticed that the scores related to physical health the SF-36 affected of these women more than the scores mental health. Con- clusion: the life quality is injured in these patients, also being aggravated by some sociodemographic, clinical and lifestyle variables. Nursing care is indispensable to face the difficulties and adherence to the treatment.}

Descriptors: Life quality; hemodialysis hospital units; nursing care; nephrology nursing. 


\section{Introdução}

A Doença Renal Crônica (DRC) é um problema de saúde pública, cada vez mais frequente, sua evolução relaciona-se a altas taxas de morbimortalidade, tanto no Brasil como no mundo ${ }^{1-2}$. Quando mais avançada, a doença denomina-se Insuficiência Renal Crônica(IRC) ${ }^{3}$, sendo necessário terapia renal substitutiva(TRS). Pode apresentar como complicações anemia, acidose metabólica, desnutrição, falência funcional renal(FFR) e até o óbito ${ }^{1}$, além disso, entram no grupo de risco hipertensos, diabéticos, idosos, pacientes com doença cardiovascular; familiares de pacientes portadores de DRC e pacientes em uso de medicações nefrotóxicas ${ }^{1-2}$.

No Brasil estima-se que o número de pacientes com DRC aumentou nos últimos anos ${ }^{4}$, consequentemente o aumento da incidência e prevalência da FFR também é cada vez mais frequente, o prognóstico não é bom e os custos com o tratamento são elevados, fazendo com que a taxa de mortalidade anual seja considerada significativa ${ }^{1,5}$.

Prova disso, é que através de dados do Censo Brasileiro de Diálise realizado anualmente pela Sociedade Brasileira de Nefrologia (SBN), houve um aumento gradual de pacientes em tratamento dialítico. Em 2000 haviam 42.695 pacientes, em 2011, 91.314 e em 2014 a quantidade de pacientes já atingia 112.004. Entretanto, essas informações precisam ser interpretadas com cautela, pois talvez nem todos os centros dialíticos brasileiros tenham respondido ao censo ${ }^{6}$.

$\mathrm{O}$ aumento da prevalência da DRC, pode estar relacionada ao aumento de doenças crônicas não transmissíveis (DCNT) como diabetes mellitus(DM) e hipertansão(HAS) ${ }^{7}$. Assim, o investimento no controle dos fatores de risco vem se tornando o grande alvo nas últimas décadas, não somente no Brasil, mas também mundialmente ${ }^{2}$. Além disso, a detecção precoce e tratamento adequado são importantes para evitar as complicações.

Quanto às TRS, uma das formas de tratamento é a hemodiálise, que realiza o processo de filtragem e depuração do sangue através de uma máquina, além de controlar a pressão arterial. É eficaz, entretanto, acarreta uma série de modificações e limitações no estilo de vida dos pacientes ${ }^{2}$, ${ }^{8-10}$. Além disso, segundo pesquisas faltam vagas para os indivíduos que necessitam fazer hemodiálise em clínicas cobertas pelo sistema público de saúde e o custo do tratamento em clínicas particulares não é acessível a todos, o que faz com que muitos não realizem o tratamento regularmente $\mathrm{e}$ aumente a taxa de mortalidade. Entretanto, a hemodiálise ainda é a modalidade mais comum de TRS em todo o mundo o,11-12.

$\mathrm{O}$ tratamento hemodialítico associado à DRC pode causar impacto negativo para o paciente e para seus familiares. Acarreta limitações ao paciente e cria a dependência de outras pessoas para a realização de atividades da vida diária, precisando de apoio para superarem a nova realidade a qual se deparam. Os cuidadores também precisam se adaptar à sobrecarga de trabalho e à responsabilidade de cuidar de uma outra pessoa com amplas necessidades ${ }^{13}$. Dessa forma, percebe-se que a DRC e o tratamento hemodialítico influenciam na percepção que os indivíduos têm de si mesmo, da posição que exercem na vida e também do contexto em que estão inseridos, ou seja, afetam a qualidade de vida (QV) desses pacientes ${ }^{14}$.

Qualidade de vida essa que é entendida por muitos estudiosos como sinônimo de saúde, bem-estar, felicidade e satisfação pessoal, entretanto corresponde a um conceito muito mais amplo, subjetivo e abrangente, que não leva em consideração somente o indivíduo, mas também todo o ambiente em que ele está inserido e as circunstâncias em que vive ${ }^{14-16}$.

Assim, a Enfermagem tem um papel importante no cuidado desses pacientes, tanto no âmbito educacional, ajudando na adesão ao tratamento, estimulando e orientando sobre o autocuidado, ou ainda, no enfrentamento das mudanças físicas e psicológicas, como também na assistência e prestação de cuidados aos mesmos, garantindo que o paciente alcance o seu bem-estar. Também é significativa no auxílio aos familiares e acompanhantes ${ }^{11,19}$.

É nesse contexto que se propõe este estudo, especificando as mulheres como público alvo, com o intuito de verificar como elas conciliam as suas atividades diárias de mães, esposas, donas de casa e trabalhadoras formais com a doença e o tratamento contínuo, principalmente nos tempos atuais em que as mulheres estão cada vez mais inseridas no mercado de trabalho. Estudar sobre o tema permite adquirir maior conhecimento e 
fornece subsídios para auxiliar no aprimoramento da assistência de enfermagem a esses pacientes, além do planejamento de políticas que permitam o atendimento mais humanizado, visando uma melhor QV.

Objetivou-se, portanto, avaliar a qualidade de vida de mulheres renais crônicas, em tratamento hemodialítico, em um Hospital Universitário do Triângulo Mineiro (MG), caracterizando a amostra quanto às variáveis sociodemográficas, clínicas e hábitos de vida, além de correlacionar a qualidade de vida com essas variáveis e também analisar a qualidade da assistência de Enfermagem prestada a essa população.

\section{Método}

Trata-se de uma pesquisa do tipo descritiva, transversal com abordagem quantitativa, realizada no setor de Hemodiálise de um Hospital Universitário do Triângulo Mineiro - MG, onde foram avaliadas todas as mulheres, em tratamento hemodialítico no período da coleta de dados e que preenchiam os critérios de inclusão, ou seja, sexo feminino, maior de 18 anos, portadoras de doença renal crônica e que estavam em tratamento no referido setor, totalizando, portanto, 19 mulheres.

A coleta de informações da pesquisa foi realizada a partir de um instrumento sociodemográfico e hábitos de vida semiestruturado, abordando as variáveis idade, cor da pele, escolaridade, estado civil, se residiam sozinhas, ocupação, obesidade, prática de atividade física, etilismo e tabagismo. Também utilizou o instrumento SF-36 (Medical Outcomes Study 36 - Item Short - Form Health Survey) que avalia a QV, com 36 itens, sendo divididos em 8 domínios: capacidade funcional (10 itens), aspectos físicos (4), dor (2), vitalidade (4), estado geral de saúde (5), aspectos sociais (2), aspectos emocionais (3) e saúde mental (5 itens), e uma questão que compara a condição de saúde atual com a de um ano atrás. A escala de escore total do instrumento varia de
0 a 100 , sendo que os escores mais altos representam melhores condições de saúde. Trata-se de um instrumento de fácil aplicação e compreensão ${ }^{18}$. Este instrumento foi complementado por questões referentes à assistência de enfermagem, com o intuito de verificar como as mulheres avaliavam a assistência recebida, as informações passadas para elas pela equipe de enfermagem do referido local em que a pesquisa foi realizada e se essa assistência ajudava no enfrentamento das dificuldades do dia a dia.

A coleta dos dados foi realizada nos meses de setembro e outubro de 2015, após aprovação pelo Comitê de Ética em Pesquisa (CEP), protocolo $\mathrm{n}^{\circ}$ 1.213.009 (CAAE $\mathrm{n}^{\circ}$ 45868115.7.0000.5152), de acordo com a Resolução 466/12 do Conselho Nacional de Saúde (CNS). As questões que nortearam este estudo foram: "As mulheres sob processo hemodialítico têm prejuízo em sua qualidade de vida?" e "A assistência de Enfermagem pode auxiliar no tratamento e enfrentamento das dificuldades dos renais crônicos em processo de hemodiálise?”.

A participação das entrevistadas foi condicionada à assinatura do Termo de Consentimento Livre e Esclarecido (TCLE), o qual autorizou a coleta de dados e a utilização dos mesmos, ficando as participantes livres para desistirem da pesquisa a qualquer momento. A entrevista teve início com a aplicação do questionário sociodemográfico, posteriormente, houve a aplicação do questionário SF-36, complementado por questões referentes à Assistência de Enfermagem. Os dados foram analisados a partir dos métodos estatísticos de correlação de Spearman e teste T- Student e ANOVA para variáveis com mais de duas opções de resposta, foi utilizado o software SPSS v.18.0.

\section{Resultados}

As características sociodemográficas, clínicas, econômicas e os hábitos de vida apresentados pelas mulheres que participaram do estudo estão presentes na tabela 1. 
Tabela 1 - Distribuição das 19 participantes segundo as variáveis sociodemográficas, clínicas, econômicas e hábitos de vida. Triangulo Mineiro (MG), 2015.

\begin{tabular}{|c|c|c|c|c|c|}
\hline \multicolumn{6}{|c|}{ Dados sociodemográficos, econômicos e hábitos de vida } \\
\hline Faixa etária & $\mathbf{N}$ & $\%$ & Estado Civil & $\mathbf{N}$ & $\%$ \\
\hline 21-30 anos & 2 & $10,53 \%$ & Solteira & 7 & $36,83 \%$ \\
\hline $31-40$ anos & 4 & $21,05 \%$ & Casada & 8 & $42,11 \%$ \\
\hline $41-50$ anos & 3 & $15,79 \%$ & Viúva & 2 & $10,53 \%$ \\
\hline $51-60$ anos & 5 & $26,31 \%$ & Separada & 2 & $10,53 \%$ \\
\hline $61-70$ anos & 3 & $15,79 \%$ & Cor da pele & & \\
\hline $71-80$ anos & 2 & $10,53 \%$ & Branca & 5 & $26,31 \%$ \\
\hline Idoso & & & Não branca & 14 & $73,69 \%$ \\
\hline Sim & 5 & $26,31 \%$ & Nível de Escolaridade & & \\
\hline Não & 14 & $73,3 \%$ & Não Alfabetizada & 2 & $10,53 \%$ \\
\hline Profissão & & & Ensino Fund. Incompleto & 8 & $42,11 \%$ \\
\hline Doméstica & 2 & $10,53 \%$ & Ensino Fund. Completo & 3 & $15,79 \%$ \\
\hline Do lar & 10 & $52,63 \%$ & Ensino med. Incompleto & 1 & $5,26 \%$ \\
\hline Aposentada & 4 & $21,05 \%$ & Ensino med. Completo & 5 & $26,31 \%$ \\
\hline Cabelereira & 1 & $5,26 \%$ & Pratica exercício físico & & \\
\hline Costureira & 1 & $5,26 \%$ & Sim & 3 & $15,79 \%$ \\
\hline Cozinheira & 1 & $5,26 \%$ & Não & 16 & $84,21 \%$ \\
\hline Religião & & & Obesidade & & \\
\hline Católica & 11 & $57,9 \%$ & Sim & 5 & $26,3 \%$ \\
\hline Evangélica & 6 & $31,53 \%$ & Não & 14 & $73,7 \%$ \\
\hline Espírita & 2 & $10,53 \%$ & Etilista & & \\
\hline Reside sozinho & & & Sim & 0 & 0 \\
\hline Sim & 1 & $5,26 \%$ & Não & 19 & $100 \%$ \\
\hline \multirow[t]{3}{*}{ Não } & 18 & $94,74 \%$ & Tabagista & & \\
\hline & & & Sim & 2 & $10,53 \%$ \\
\hline & & & Não & 17 & $89,47 \%$ \\
\hline
\end{tabular}

Fonte: próprio autor.

Em relação à doença de base houve um significativo destaque para HAS com 11 mulheres $(57,89 \%)$, seguida de DM com 5 mulheres $(26,31 \%)$, isoladas ou associadas a outras patologias. Outras causas manifestadas pelas entrevistadas foram cistinose, bexiga neurogênica, uso prolongado de Sonda Vesical de Demora, lúpus eritematoso, hidronefrose, infecção de urina e nefrite, em porcentagens menores, além de uma paciente que não soube informar a cau- sa. Dentre as mulheres estudadas, sete $(36,84 \%)$ não estavam na lista de espera pelo transplante e as outras doze mulheres $(63,15 \%)$ esperavam pela cirurgia.

A partir da análise do instrumento SF-36 foi possível verificar a média recebida em cada domínio, demonstrando quais aspectos avaliados foram mais influenciados pela DRC. Os domínios com os maiores escores representam melhor QV, conforme pode ser verificado na tabela 2 . 
Tabela 2 - Valores médios das dimensões do SF-36 entre as 19 participantes com DRC participantes da pesquisa. Triângulo Mineiro (MG), 2015.

\begin{tabular}{cccccc}
\hline Domínio & N & Mínimo & Máximo & Média & Desvio padrão \\
\hline Capacidade Funcional & 19 & 0 & 100 & 57,63 & 32,032 \\
Aspectos físicos & 19 & 0 & 100 & 19,74 & 32,892 \\
Dor & 19 & 0 & 100 & 57,16 & 30,902 \\
Estado geral de saúde & 19 & 12 & 95 & 60,05 & 28,941 \\
Vitalidade & 19 & 30 & 100 & 68,16 & 20,897 \\
Aspectos sociais & 19 & 13 & 100 & 71,05 & 32,822 \\
Aspectos emocionais & 19 & 0 & 100 & 47,36 & 44,882 \\
Saúde mental & 19 & 28 & 100 & 64,63 & 29,159
\end{tabular}

Fonte: próprio autor.

Já na tabela 3 encontra-se os coeficientes de correlação de Sperman entre as dimensões do
SF-36 e as variáveis clínicas para as 19 mulheres estudadas.

Tabela 3 - Correlação entre as variáveis do SF-36 e as variáveis clínicas, segundo teste de correlação de Spearman. Triângulo Mineiro (MG), 2015.

\begin{tabular}{ccccccccc}
\hline $\begin{array}{c}\text { Variáveis } \\
\text { Clínicas } \\
\text { SF-36 }\end{array}$ & $\begin{array}{c}\text { Cap. } \\
\text { Func. }\end{array}$ & $\begin{array}{c}\text { Aspec. } \\
\text { Fís. }\end{array}$ & Dor & $\begin{array}{c}\text { Est. } \\
\text { Geral } \\
\text { Saúde }\end{array}$ & Vital. & $\begin{array}{c}\text { Aspec. } \\
\text { Soc. }\end{array}$ & $\begin{array}{c}\text { Aspec. } \\
\text { Emoc. }\end{array}$ & $\begin{array}{c}\text { Saúde } \\
\text { Mental }\end{array}$ \\
\hline $\begin{array}{c}\text { Idade } \\
\text { IMC }\end{array}$ &,- 336 &,$- 497^{\star}$ &,- 250 &,- 141 &,- 095 &,- 022 &,- 305 &,- 095 \\
$\begin{array}{c}\text { Tempo de Diagnóstico } \\
\text { da DRC }\end{array}$ &, 003 &,- 388 &, 040 &,- 168 &,- 338 &, 159 &,- 283 &,- 152 \\
&, 014 &, 310 &,- 363 &, 146 &, 143 &, 069 &, $\mathbf{4 9 9 *}$ &, 273
\end{tabular}

${ }^{*}$ Correlação significativa ao nível de p-valor igual ou menor que 0,05 . Fonte: próprio autor.

Legenda do SF-36:

- Cap. Func. - Capacidade Funcional

- Aspec. Fís. - Aspectos físicos

- Est. Geral Saúde - Estado geral de saúde

- Vital. - Vitalidade

- Aspec. Soc. - Aspectos sociais

- Aspec. Emoc. - Aspectos Emocionais

Na tabela 4 são mostrados os escores médios obtidos para as variáveis sociodemográficas, clí- nicas e hábitos de vida em relação a cada uma das dimensões do SF-36.

Tabela 4 - Escores médios das dimensões do SF-36, segundo as variáveis sociodemográficas, clínicas e hábitos de vida. Triângulo Mineiro (MG), 2015.

\begin{tabular}{|c|c|c|c|c|c|c|c|c|c|c|c|c|c|c|c|c|}
\hline Obesidade & \multicolumn{2}{|c|}{$\begin{array}{c}\text { Capacidade } \\
\text { Funcional }\end{array}$} & \multicolumn{2}{|c|}{$\begin{array}{l}\text { Aspectos } \\
\text { Físicos }\end{array}$} & \multicolumn{2}{|c|}{ Dor } & \multicolumn{2}{|c|}{$\begin{array}{c}\text { Estado } \\
\text { Geral } \\
\text { Saúde }\end{array}$} & \multicolumn{2}{|c|}{ Vitalidade } & \multicolumn{2}{|c|}{$\begin{array}{l}\text { Aspectos } \\
\text { Sociais }\end{array}$} & \multicolumn{2}{|c|}{$\begin{array}{c}\text { Aspectos } \\
\text { Emocio- } \\
\text { nais }\end{array}$} & \multicolumn{2}{|c|}{$\begin{array}{l}\text { Saúde } \\
\text { Mental }\end{array}$} \\
\hline Obeso & 56,0 & 68,5 & 10,0 & ,355 & 49,2 & 92,9 & 45,6 & 49,2 & 58,0 & ,357 & 70,0 & ,191 & 46,6 & ,771 & 58,4 & ,342 \\
\hline Não Obeso & 58,2 & & 23,2 & & 60,0 & & 65,2 & & 71,7 & & 71,4 & & 47,6 & & 66,8 & \\
\hline \multicolumn{17}{|l|}{ Idoso } \\
\hline Sim & 51,0 & ,179 & ,00 & $013^{*}$ & 41,0 & 997 & 47,8 & ,576 & 63,0 & ,486 & 50,0 & ,410 & 33,3 & , 107 & 60,8 & ,254 \\
\hline Não & 60,0 & & 26,7 & & 62,9 & & 64,4 & & 70,0 & & 78,5 & & 52,3 & & 66,0 & \\
\hline
\end{tabular}




\begin{tabular}{|c|c|c|c|c|c|c|c|c|c|c|c|c|c|c|c|c|}
\hline \multicolumn{17}{|l|}{$\begin{array}{c}\text { Reside } \\
\text { sozinho }\end{array}$} \\
\hline Sim & 45,0 & ,697 &, 00 &, 553 &, 00 &, 054 & 82,0 & ,452 & 75,0 & ,747 & 100 & ,380 & 100,0 & ,239 & 100, & ,222 \\
\hline Não & 58,3 & & 20,8 & & 60,3 & & 58,8 & & 67,7 & & 69,4 & & 44,4 & & 62,6 & \\
\hline \multicolumn{17}{|l|}{ Cor } \\
\hline Branco & 78,0 &, $042^{*}$ & 40,0 & ,226 & 78,6 &, $042^{*}$ & 78,2 &, $026^{*}$ & 80,0 &, $035^{*}$ & 80,0 & ,450 & 73,3 &, 118 & 93,6 &, $000^{*}$ \\
\hline Não branco & 50,3 & & 12,5 & & 49,5 & & 53,5 & & 63,9 & & 67,8 & & 38,0 & & 54,2 & \\
\hline \multicolumn{17}{|l|}{ Tabagismo } \\
\hline Sim & 57,5 & 998 & 12,5 & ,643 & 76,5 & ,056 & 61,0 & ,978 & 60,0 & ,812 & 81,2 & ,314 & 33,3 & ,720 & 30 &, $000^{*}$ \\
\hline Não & 57,6 & & 20,5 & & 54,8 & & 59,9 & & 69,1 & & 69,8 & & 49,0 & & 68,7 & \\
\hline \multicolumn{17}{|l|}{$\begin{array}{c}\text { Prat. } \\
\text { Atividade } \\
\text { Física }\end{array}$} \\
\hline Sim & 71,6 &, 559 & 25,0 & ,497 & 62,3 & ,670 & 85,3 &, $016^{*}$ & 70,0 & ,864 & 83,3 & ,346 & 11,1 &, $029^{*}$ & 49,3 & ,224 \\
\hline Não & 55,0 & & 18,7 & & 56,1 & & 55,3 & & 67,8 & & 68,7 & & 54,1 & & 67,5 & \\
\hline
\end{tabular}

${ }^{\star}$ Estatisticamente significante para $\mathrm{p}<0,05$. Continua.

\begin{tabular}{|c|c|c|c|c|c|c|c|c|c|c|c|c|c|c|c|c|}
\hline $\begin{array}{l}\text { Variáveis } \\
\text { Estado Civil }\end{array}$ & \multicolumn{2}{|c|}{$\begin{array}{l}\text { Capacidade } \\
\text { Funcional }\end{array}$} & \multicolumn{2}{|c|}{$\begin{array}{l}\text { Aspectos } \\
\text { físicos }\end{array}$} & \multicolumn{2}{|c|}{ Dor } & \multicolumn{2}{|c|}{$\begin{array}{l}\text { Estado } \\
\text { geral de } \\
\text { saúde }\end{array}$} & \multicolumn{2}{|c|}{ Vitalidade } & \multicolumn{2}{|c|}{$\begin{array}{l}\text { Aspectos } \\
\text { sociais }\end{array}$} & \multicolumn{2}{|c|}{$\begin{array}{l}\text { Aspectos } \\
\text { emocionais }\end{array}$} & \multicolumn{2}{|c|}{$\begin{array}{l}\text { Saúde } \\
\text { Mental }\end{array}$} \\
\hline Casada & 68,1 & ,279 & 25,0 & ,647 & 69,3 & ,298 & 57,2 & ,835 & 65,0 & 608 & 68,7 & ,653 & 79,1 &, $034^{*}$ & 75,0 & ,308 \\
\hline Solteira & 61,4 & & 25,0 & & 49,1 & & 61,7 & & 65,7 & & 67,8 & & 19,0 & & 48,0 & \\
\hline Viúva & 32,5 & & - & & 66,5 & & 76,0 & & 87,5 & & 62,5 & & 16,6 & & 78,0 & \\
\hline Separada & 27,5 & & - & & 27,0 & & 49,5 & & 70,0 & & - & & 50,0 & & 68,0 & \\
\hline \multicolumn{17}{|c|}{ Escolaridade } \\
\hline Analfabeto & 15,0 & ,070 & - &, $038^{\star}$ & 58,0 &, 321 & 47,0 & ,501 & 75,0 &, 817 & 87,5 & ,161 & - & ,323 & 52,0 & ,260 \\
\hline $\begin{array}{l}\text { Ens. Fund. } \\
\text { Incompleto }\end{array}$ & 46,8 & & 9,3 & & 54,5 & & 63,5 & & 66,2 & & 87,5 & & 45,8 & & 70,5 & \\
\hline $\begin{array}{l}\text { Ens. Fund. } \\
\text { Completo }\end{array}$ & 70,0 & & 8,3 & & 30,6 & & 39,6 & & 56,6 & & 37,5 & & 33,3 & & 33,3 & \\
\hline $\begin{array}{l}\text { Ens. Med. } \\
\text { Completo }\end{array}$ & 78,0 & & 35,0 & & 68,4 & & 65,0 & & 73,0 & & 62,5 & & 66,6 & & 74,4 & \\
\hline \multicolumn{17}{|l|}{$\begin{array}{c}\text { Espera } \\
\text { Transplante }\end{array}$} \\
\hline Sim & 73,3 &, $007^{*}$ & 31,2 &, $014^{*}$ & 58,0 & ,876 & 68,3 &, 112 & 71,2 & ,451 & 71,8 & ,887 & 55,5 &, 285 & 71,0 & ,225 \\
\hline Não & 30,7 & &, 00 & & 55,5 & & 45,8 & & 62,8 & & 69,6 & & 33,3 & & 53,7 & \\
\hline \multicolumn{17}{|l|}{ Religião } \\
\hline Católica & 43,1 &, $004^{*}$ & 6,82 &, $013^{*}$ & 43,7 &, 051 & 52,3 &, $033^{*}$ & 64,0 & ,363 & 69,3 & ,343 & 33,3 &, 243 & 59,6 & ,398 \\
\hline Evangélica & 90,0 & & 50,0 & & 70,5 & & 83,0 & & 78,3 & & 83,3 & & 61,1 & & 78,0 & \\
\hline Espírita & 40,0 & & - & & 91,0 & & 33,5 & & 60,0 & & 43,7 & & 83,3 & & 52,0 & \\
\hline
\end{tabular}

${ }^{\star}$ Estatisticamente significante para $\mathrm{p}<0,05$.

Fonte: próprio autor.

E na Tabela 5 estão apresentadas as Enfermagem e ajuda da mesma durante o informações quanto à Assistência de tratamento hemodialítico. 
Tabela 5 - Distribuição das respostas segundo a qualidade da assistência de Enfermagem e das informações passadas às pacientes. Triângulo Mineiro (MG), 2015.

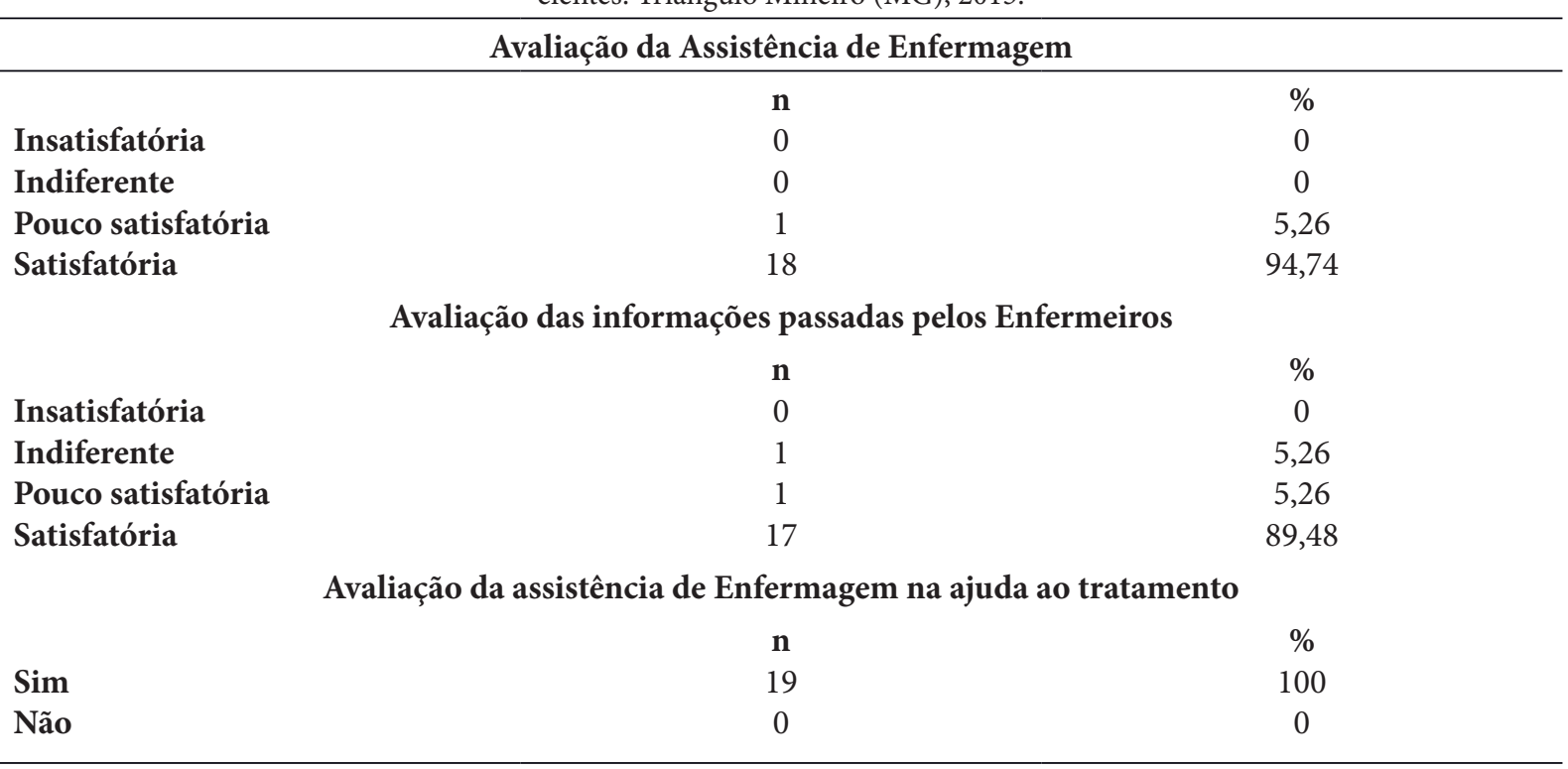

Fonte: próprio autor.

\section{Discussão}

Em relação a faixa etária, conforme pode ser observado na tabela 1 , houve o predomínio da idade entre 51 e 60 anos. Outros estudos comprovaram o que foi identificado nesta pesquisa, em que o maior número de pacientes em tratamento hemodialítico possui entre 45 e 64 anos, idade economicamente ativa, o que afeta ainda mais a $\mathrm{QV}^{15,19,20}$.

Nos EUA a prevalência da DRC também aumenta com a idade, fazendo-se uma comparação de $7,2 \%$ em idosos entre 65 e 74 anos e $17 \%$ nos idosos com 85 anos ou mais ${ }^{2}$, já o censo da SBN de 2014 trouxe que o maior percentual de pacientes em diálise tem idade entre 19 e 64 anos correspondendo a $66,4 \%{ }^{6}$. A idade do paciente influencia na sua $\mathrm{QV}$, pois com o avançar da idade há perda da capacidade funcional, vitalidade e aspectos sociais, também interfere na aceitação e percepção da doenç $\mathrm{a}^{9,19-20}$, além do aumento de comorbidades, agravando o estado físico e mental dos mesmos.

Um estudo espanhol mostra que os pacientes mais jovens apresentam melhor estado funcional quando comparado com os idosos, e que a presença de comorbidades diminui a QV quando comparada a população em geral $^{21}$. Já em relação à aceitação da doença, essa acontece ao longo do tempo de tratamento, quando as pessoas se tornam capazes de se adaptar à nova realidade ${ }^{20}$.

Outro fator marcante, é que o tratamento, debilitante e contínuo, impede as mulheres de exercerem suas funções de trabalho, há o desenvolvimento de sentimentos de inutilidade, podendo inclusive acarretar em problemas psicológicos, como depressão ${ }^{11,22-23}$. Estudos na Espanha também trazem que muitas vezes a hemodiálise é iniciada tardiamente, isso associado a comorbidades, incapacidade para o pagamento da diálise e a dificuldade de deslocamento para os centros hemodialíticos levam ao aumento da taxa de mortalidade ${ }^{21}$.

Também há prejuízo das viagens e atividades de lazer, afetando ainda mais a QV desses pacientes ${ }^{22-23}$. Por tratar-se de um evento traumático, os pacientes se apegam a fé e religião para tentarem superar ou pelo menos aceitar a realidade em que se encontram ${ }^{8}$, como também foi evidenciado a partir da tabela 4 , sendo que a maioria das mulheres $(57,9 \%)$ disseram ser católicas. Além da religião, os pacientes buscam ajuda em seus parceiros, familiares e amigos ${ }^{8,19,24}$.

Segundo estudo colombiano, a religião auxilia na adaptação a situações de diagnóstico e tratamento. Permite ao paciente melhor lidar com a 
dor e gerir seus sentimentos, além de promover apoio psicológico ${ }^{25}$. Estudos também demonstram que os pacientes em tratamento hemodialítico buscam apoio em seus familiares. Segundo trabalho mexicano, a família continua sendo a principal fonte de apoio encontrada pelos pacientes em TRS, indo de acordo com um estudo chileno que evidencia que as pessoas com DRC que vivem sozinhas apresentaram escores mais baixos quando tiveram sua QV avaliada ${ }^{20}$.

Quanto à escolaridade, sabe-se que quanto maior o nível escolar, maiores são as informações obtidas para a prevenção de agravos e adoção de hábitos de vida saudáveis ${ }^{21}$, entretanto no estudo em questão, percebeu-se maior número de mulheres que possuíam apenas o ensino fundamental incompleto, como está ilustrado na tabela 1 , concordando com um estudo espanhol que apresentava porcentagem significativa de renais crônicos em hemodiálise com baixo nível de instrução ${ }^{20}$.

Assim, foi possível perceber que a maioria das variáveis sociodemográficas trabalhadas no estudo influenciaram na QV dessas mulheres.

Em relação aos hábitos de vida, estudos demonstram a importância da prática de atividade física já que contribui para o controle pressórico, aumenta a capacidade funcional, força e resistência muscular, melhora a função cardíaca e pode ainda aumentar significativamente a remoção de ureia, creatinina e potássio, melhorando a eficiência da hemodiálise e reduzindo cerca de 30 minutos da duração das sessões, entretanto somente 3 mulheres relataram praticar atividade física neste estudo ${ }^{26}$.

O etilismo, tabagismo e o peso também merecem destaque, já que são fatores agravantes para a IRC $^{27}$. Apesar de a minoria das mulheres estudadas possuírem Índice de Massa Corporal(IMC) maior que $30 \mathrm{~kg} / \mathrm{m}^{2}$, sabe-se que a obesidade geralmente está associada à DM e HAS, que são as principais doenças de base da DRC, correspondendo a um fator de risco para o desenvolvimento de lesão glomerular e progressão para $\mathrm{FFR}^{27}$. Dessa forma, investir no controle pressórico é importante na redução da DRC e outras complicações ${ }^{28}$.

No que diz respeito a doença de base da DRC, há um destaque para a HAS e DM tanto no Brasil como em outros países, como Espanha, Sri Lanka e Cuba, sendo que na Nigéria e na África Subsaariana, predomina a glomerulonefrite crô- nica e na América Latina, o DM se destaca, principalmente em Porto Rico, México e Colômbia ${ }^{21}$.

Em relação a QV, a mesma diz respeito a percepção que o indivíduo tem da sua própria vida, no contexto cultural em que vive ${ }^{3,15}$. Assim, a condição crônica da doença, as mudanças na imagem corporal, restrições líquidas e dietéticas, convívio com uma doença incurável, tratamento rigoroso e contínuo e a utilização de medicamentos, além da dependência de uma máquina correspondem a permanentes fontes de estresse ${ }^{15,18,19,22,29}$. Entretanto, apesar de o tratamento dialítico ser considerado fator limitador para o paciente, ele também pode ser potencializador, já que consegue prolongar a vida do cliente e melhorar o seu quadro de saúde ${ }^{19,23}$.

Diante disso e com a avaliação da QV destas mulheres foi possível perceber a partir da tabela 2 que as dimensões que apresentaram maiores escores médios foram: aspectos sociais, vitalidade, saúde mental, estado geral de saúde e os que apresentaram menores escores foram: aspectos físicos, aspectos emocionais, dor e capacidade funcional, ou seja, os domínios referentes à saúde física afetaram mais a QV das mulheres do estudo do que os domínios de saúde mental. Resultado parecido foi observado em outros estudos ${ }^{3,9,17}$. Na dimensão física, percebe-se limitação devido a deterioração musculoesquelética e fraquezas comuns ocasionadas pela hemodiálise, inclusive as alterações eletrolíticas ${ }^{11,19}$. Estudo chileno também trouxe que o fato de os aspectos físicos serem mais afetados, reflete a capacidade que os pacientes têm de se adaptarem psicologicamente a situação em que se encontram, principalmente ao longo do tempo ${ }^{20}$.

Com relação ao estado de saúde emocional, os pacientes geralmente apresentam alterações de humor que dependem da negação ou aceitação do mesmo em relação a sua situação. Essa alteração também está relacionada com o acúmulo ou retirada exagerada de líquidos do organismo durante as sessões de hemodiálise podendo causar até mesmo depressão. Outros problemas psicológicos podem ser encontrados, nos pacientes e seus familiares ${ }^{19}$. Quanto aos aspectos sociais, os problemas enfrentados são timidez, preconceito, falta de emprego, de apoio familiar e de atividades de lazer. Por isso o fortalecimento da rede de apoio familiar e social é de extrema importância para confortar o paciente e ajudá-lo a superar as dificuldades e limitações pessoais e sociais ${ }^{19}$. 
Já partir da correlação de Sperman, demonstrada na tabela 3, foi possível relacionar as variáveis do instrumento SF-36 com variáveis idade e tempo de diagnóstico da DRC. Essa correlação permitiu analisar que idade e aspectos físicos são inversamente proporcionais, ou seja, quanto maior a idade, menor será o escore recebido no domínio aspecto físico, portanto maior será o comprometimento da QV relacionado a esse domínio, o que também pode ser confirmado por outros estudos que relatam que os idosos tiveram piores escores nos domínios abordados pelo instrumento em questão $0^{2,9-10,19}$.

Outra correlação foi entre aspectos emocionais e tempo de diagnóstico, que são diretamente proporcionais, demonstrando que quanto menor o tempo de diagnóstico menor será o escore recebido no domínio aspectos emocionais, fato também discutido anteriormente e demonstrado em outros estudos que relatam que a insegurança e o desconforto são mais frequentes no início da terapêutica, pois com o tempo os pacientes se habituam e adaptam-se a nova realidade ${ }^{19-20}$.

Já em relação aos escores médios das dimensões do SF-36, segundo as variáveis sociodemográficas, clínicas e hábitos de vida ilustrados na tabela 4, percebeu-se que, os não obesos apresentaram valores médios dos escores maiores que os obesos em todos os domínios. A obesidade tornou-se um problema de saúde pública e afeta a $\mathrm{QV}$, não apenas em relação a DRC, mas também causa uma série de outras complicações como: distúrbios cardiovasculares, endócrinos, respiratórios, gastrointestinais, dermatológicos, geniturinários, músculoesqueléticos, distúrbios psicossociais; neoplasias, entre outras ${ }^{27}$. Segundo o censo da SBN que avaliou o IMC, constatou-se que 10\% dos pacientes estavam desnutridos, $53 \%$ normais e $37 \%$ em sobrepeso, obesidade ou obesidade mórbida $^{6}$, já em um estudo espanhol 59\% dos pacientes avaliados estavam com sobrepeso ${ }^{21}$.

Quanto à idade, os não idosos tiveram escores maiores em todos os domínios do SF-36, possuindo melhor QV, além de apresentar diferença estatisticamente significativa no domínio aspectos físicos. Já as mulheres de cor da pele não branca apresentaram menores escores em todos os domínios do SF-36 em relação àquelas de cor da pele branca, com diferença estatisticamente significan- te nos domínios capacidade funcional, dor, estado geral de saúde, vitalidade e saúde mental, o que permite dizer que para esses domínios, a QV das mulheres consideradas brancas é relevantemente melhor do que aqueles com cor da pele não branca. Em estudo norte americano é visualizado que a prevalência da DRC é maior entre os negros, em aproximadamente $50 \%$ em relação aos brancos, já em asiáticos, a prevalência é em torno de $11 \%{ }^{2}$.

Analisando ainda os dados da tabela 4, a realização de atividade física entre as mulheres colaborou para a QV, exceto nos domínios aspectos emocionais e saúde mental em que aqueles que não praticam atividades físicas receberam maiores escores, esse fato discorda de outros trabalhos publicados, e estatisticamente pode-se falar que houve diferença significante nos domínios estado geral de saúde e aspectos emocionais ${ }^{26}$.

Quanto ao tabagismo, as mulheres não fumantes apresentaram escores menores para os domínios dor, estado geral de saúde e aspectos sociais, quando comparadas com as mulheres fumantes, tiveram valores estatisticamente significantes somente para o domínio saúde mental, essa informação vai de acordo com um estudo chileno que traz que os fumantes em tratamento hemodialítico apresentam melhor $\mathrm{QV}^{20}$. Quanto a instrução, quanto maior a escolaridade, melhor a $\mathrm{QV}$, conforme citado anteriormente e também concordando com outros estudos, porém nos domínios vitalidade e aspectos sociais, os escores maiores foram para as analfabetas ${ }^{20}$. Já a espera pelo transplante também influencia positivamente na $\mathrm{QV}$, indicando a expectativa da paciente por uma melhor condição de vida associada a realização da cirurgia. Os domínios capacidade funcional e aspectos físicos apresentaram diferenças estatisticamente significantes ${ }^{20}$. Em julho de 2014, de acordo com o censo da SBN, haviam 31.499 pacientes na fila de espera do transplante, cerca de $29 \%$ dos indivíduos $^{6}$. Estudos fora do Brasil indicam que o transplante renal é o tratamento mais rentável para a doença em estágio final, além de garantir economia e $\mathrm{QV}^{20}$.

Em relação a assistência de enfermagem, solicitou-se às mulheres que participaram da pesquisa que avaliassem a assistência recebida no setor em que faziam tratamento e se a mesma era benéfica ou não para a realização de hemodiálise e 
consequente convívio com a DRC. A avaliação foi realizada a partir da classificação dos cuidados recebidos e das informações passadas em insatisfatório, indiferente, pouco satisfatório ou satisfatório. Ao analisar as respostas obtidas, presentes na tabela 5, encontrou-se uma contradição, já que uma mulher considerou a assistência de enfermagem que recebia como pouco satisfatória e em relação às informações passadas pelos enfermeiros, uma considerou como insatisfatória e uma como pouco satisfatória, mas quando tiveram que avaliar se a assistência de enfermagem ajudava no tratamento, nas dificuldades do dia a dia e no convívio com a doença incurável, todas disseram que sim. A contradição encontrada nas respostas mostra como alguns pacientes apresentam certa dificuldade para compreender adequadamente as questões solicitadas nos instrumentos de coleta de dados e por vezes acabam respondendo contraditoriamente, talvez por falta de real entendimento.

Assim, pode-se dizer que a assistência de enfermagem, de forma geral, foi avaliada como sendo de extrema importância durante o tratamento hemodialítico, mesmo porque, por tratar-se de pacientes críticos, os renais crônicos necessitam de um tratamento especializado, com profissionais que possuam conhecimento para prestar uma assistência adequada. Assim, a função do enfermeiro não é apenas executar técnicas e procedimentos, mas desenvolver vínculo, habilidades de comunicação, apoio e empatia, satisfazendo as necessidades dos pacientes e de seus familiares ${ }^{11,19}$.

A pesquisa em questão teve como limitação a população reduzida quando comparada a outros estudos já realizados, esse fato se deu tanto pela especificação do sexo como também pelo curto período de coleta de dados, porém, as informações coletadas contribuirão para o maior conhecimento sobre a QV dos renais crônicos e criação de estratégias que atendam às necessidades de cada paciente, e também ajudará na melhoria da assistência de Enfermagem prestada a eles, tornando-a mais integral e humanizada.

\section{Conclusões}

Percebeu-se que a QV relacionada à saúde de mulheres em tratamento hemodialítico sofre impacto, principalmente nos domínios aspectos físicos, aspectos emocionais, dor e capacidade funcional, causando limitação física e psicológica, além disso, algumas variáveis sociodemográficas, clínicas e hábitos de vida correlacionam-se com a QV podendo comprometê-la.

Dentre as variáveis estudadas, destacaram-se idade; tempo de diagnóstico da DRC; cor da pele; prática de atividade física que proporciona uma melhor QV na maioria dos domínios analisados e a instrução, sendo esta diretamente proporcional com a QV.

A assistência de enfermagem também foi vista como indispensável, mesmo porque o Enfermeiro é o profissional que está em contato direto com o paciente, atendendo suas necessidades e de seus familiares, esclarecendo as dúvidas e criando vínculos, de forma individual e humanizada.

Diante disso, os resultados deste trabalho subsidiarão conhecimentos necessários para que a enfermagem desenvolva uma assistência mais adequada ao paciente hemodialítico; possibilitará o melhor planejamento de ações que atendam às necessidades individuais, além do desenvolvimento de instrumentos específicos visando alcançar uma melhor QV para esta população.

\section{Referências}

1. Bastos MG, Bregman R, Kirsztajn GM. Doença renal crônica: frequente e grave, mas também prevenível e tratável. Rev. Assoc. Med. Bras. 2009 [citado 2016 Mar 26]; 56(2): 248-253.

2. United States Renal Data System. 2014 USRDS Annual Data Report. National Institutes of Health, National Institute of Diabetes and Digestive and Kidney Diseases. Bethesda; 2014

3. Higa K, Kost MT, Soares DM, Morais MC, Polins BRG. Quality of life of patients with chronic renal insufficiency undergoing dyalisis treatment. Acta Paul. Enferm. 2008 [cited 2016 apr 22]; 21 (número especial): 203-206.

4. Crestani Filho VJ, Rodrigues RAC. Progression of chronic kidney disease: ambulatory experience in Santarém - Pará. J. Bras. Nefrol. 2013 [cited 2016 Mar 17]; 35(2): 99-106.

5. Bastos MG, Kirsztajn GM. Chronic kidney disease: importance of early diagnosis, immediate referral and structured interdisciplinary approach to improve outcomes in patients not yet on dialysis. J. Bras. Nefrol. 2011 [cited 2016 Mar 03]; 33(1): 93-108. 
6. Sesso RC, Lopes AA, Thomé FS, Lugon JR, Martins CT. Inquérito Brasileiro de Diálise Crônica 2014. J Bras Nefrol. 2014 [citado 09 ago 2017]; 38 (1):54-61.

7. Ribeiro IP, Pinheiro ALS, Soares ALA, Santos NFM. Perfil epidemiológico dos portadores de insuficiência renal crônica submetidos à terapia hemodialítica. Enferm. Foco. 2014. 5 (3/4): 65-69.

8. Valcanti CC, Chaves ECL, Mesquita AC, Nogueira DA, Carvalho EC. Religious/spiritual coping in people with chronic kidney disease undergoing hemodialysis. Rev. Esc. Enferm. USP. 2012 [citado 2016 Apr 24]; 46(8): 838-845.

9. Soares KTA, Viesser MV, Rzniski TAB, Brum EP. Eficácia de um protocolo de exercícios físicos em pacientes com insuficiência renal crônica, durante o tratamento de hemodiálise, avaliada pelo SF-36. Fisioter. Mov. 2011 [citado 2016 Mar 09]; 24(1): 133-140.

10. Orlandi FS, Pepino BG, Pavarini SCL, Santos DA, Mendiondo MSZ. The evaluation of the level of hope of elderly chronic kidney disease patients undergoing hemodialysis. Rev. Esc. Enferm. USP. 2011[cited 2016 Apr 24]; 46(4): 900-905.

11. Pabon-Varela Y, Paez-Hernandez KS, Paez-Hernandez KD, Medina-Atencio EC, López-Tavera M, Salcedo-Quintero LV. Calidad de vida del adulto con insuficiencia renal crónica, una mirada bibliográfica. Duazary. 2015 [cited 2017 Aug 26]; 12 (2):157-163.

12. Cabral AS. Carta da nefrologia. Sociedade Brasileira de Nefrologia. 2017 [citado 2017 Jul 02].

13. Angarita OMV. Percepción del apoyo social funcional en cuidadores familiares de enfermos crónicos. Aquichan. 2011 [citado 08 ago 2017]; 11(3): 274-286.

14. Pereira ÉF, Teixeira, CS, Santos A. Qualidade de vida: abordagens, conceitos e avaliação. Rev. Bras. Educ. Fís. Esporte. 2012, [citado 2018 Mar. 15]; 26(2): 241-250.

15. Alvares J, Almeida AM, Szuster DAC, Gomes IC, Andrade EIG, Acurcio FA, et al. Fatores associados à qualidade de vida de pacientes em terapia renal substitutiva no Brasil. Ciên. Saúde Coletiva. 2013[citado 2016 mar 09]; 18(7): 1903-1910.

16. Ravagnani LMB, Domingos NAM, Miyazaki MCOS. Qualidade de vida e estratégias de enfrentamento em pacientes submetidos a transplante renal. Estud. Psicol. 2007 [citado 2016 Mai 04]; 12(2): 177-184.

17. Santos I, Rocha RPF, Berardinelli LMM. Necessidades de orientação de enfermagem para o autocuidado de clientes em terapia de hemodiálise. Rev. Bras. Enferm. 2011 [citado 2016 apr 18]; 64(2): 335-342.
18. Ciconelli RM, Ferraz MB, Santos W, Meinão I, Quaresma MR. Tradução para a língua portuguesa e validação do questionário genérico de avaliação de qualidade de vida SF - 36 (Brasil SF-36). Rev. Bras. Reumatol. 1999 [citado 2016 mai. 26]; 39(3): 143-150.

19. Takemoto AY, Okubo P, Bedendo J, Carreira L. Avaliação da qualidade de vida em idosos submetidos ao tratamento hemodialítico. Rev. Gaúcha Enferm. 2011 [citado 2016 Mar 28]; 32(2): 256-262.

20. Guerra V, Sanhueza O, Cáceres M. Calidad de vida de personas en hemodiálisis crónica: relación con variables sociodemográficas, médico-clínicas y de laboratorio. Revista. Latino-Am. Enfermagem. 2012 [cited 2017 Aug 26]; 20(5): 838-846.

21. Pérez-García R, Martín-Malo A, Fort J, Cuevas X, Lladós F, Lozano J. Baseline characteristics of an incident haemodialysis population in Spain: results from ANSWER--a multicentre, prospective, observational cohort study. Nephrol Dial Transplant. 2009 [cited 2017 Aug 24];24:578-88

22. Santos TMB, Frazão IS. Qualidade de vida dos trabalhadores que realizam hemodiálise. Rev Ciênc. Méd. 2012 [citado 2016 Mai 24]; 21(1-6): 5-14.

23. Silva AS, Silveira RS, Fernandes GFM, Lunardi VL, Backes VMS. Percepções e mudanças na qualidade de vida de pacientes submetidos à hemodiálise. Rev. Bras. Enferm. 2011 [citado 2016 Jun 02]; 64(5): 839-844.

24. Terra FS, Costa AMDD. Avaliação da qualidade de vida de pacientes renais crônicos submetidos à hemodiálise. Rev. Enferm. Uerj, Alfenas. 2007; 15(3): 430-436.

25. Escobar LMV. Intervención educativa para enfermería: cuidado espiritual durante la enfermedad crónic. Aquichan. 2016 [Citado 08 ago 2017]; 17 (1):30-41.

26. Moura RMF, Silva FCR, Ribeiro GM, Sousa LA. Efeitos do exercício físico durante a hemodiálise em indivíduos com insuficiência renal crônica: uma revisão. Fisioter. Pesqui. 2008[citado 2016 Mai 06]; 15(1): 86-91.

27. Tavares TB, Nunes SM, Santos MO. Obesidade e qualidade de vida: revisão da literatura. Rev. Med. Minas Gerais. 2010 [citado 2016 Jun 09]; 20(3): 359-366.

28. Caetano ERP, Praxedes JN. Lesão renal na hipertensão essencial. HiperAtivo. 1998[citado 2016 Jun 02]; 5(4): 234-241.

29. Valle LS, Souza VF, Ribeiro AM. Estresse e ansiedade em pacientes renais crônicos submetidos à hemodiálise. Estud.Psicol. 2013 [citado 2016 Abr 29]; 30 (1): 131-138. 